\title{
Effets de divers modes de fertilisation ( $N, P, K$ ) sur certaines caractéristiques physiques, chimiques, mécaniques et propriétés papetières du pin maritime des Landes (Pinus pinaster Ait.)
}

\section{Bois de compression et propriétés papetières}

\author{
S. OHTA, R. KELLER * et G. JANIN :* \\ Forestry and Forest Products Research Institute \\ P.O. Box 16, Tsukuba Norin Kenkyu, Danchi Nai, Ibaraki j05, Japon \\ *I.N.R.A., Ecole Nationale du Génie Rural, des Eaux et des Forêts \\ Laboratoire de Recherches sur les Produits forestiers \\ 14, rue Girardet, F 54042 Nancy Cedex \\ ** I.N.R.A., Station de Recherches sur la Qualité de's Bois \\ Centre de Recherches forestières de Nancy \\ Champenoux, F 54280 Seichamps
}

\begin{abstract}
Résumé
Le gain de production en volume obtenu par la fertilisation minérale $(N, P, K)$ qui n'entraîne pas une diminution importante des propriétés mécaniques (cas de la fertilisation $\mathrm{P}+\mathrm{K}$ ou $\mathrm{P}$ ) comme on l'a vu dans la première partie (Ann. Sci. For., 40 (3), 283-298), est également appréciable lors des utilisations papetières du bois de pin maritime.

En effet, il n'existe pas de différences importantes entre lc bois témoin et lc bois fertilisé pour le rendement en pâte et la longueur des fibres.

Des observations détaillées montrent cependant que la zone de bois de compression - qui fournit par nature un rendement en pâte faible - présente un rendement en pâte plus faible $(-4,6$ p. 100) chez les arbres fertilisés que chez les arbres témoins. En revanche, les autres zones de bois normal chez les arbres fertilisés possèdent un rendement en pâte supérieur $(+0,6$ p. 100) aux zones correspondantes chez les arbres témoins.

La longueur des fibres qui, dans le jeune âge, varic très rapidement d'une année à l'autre n'est pas modifiéc par la fertilisation ni entre les années de même rang ni suivant la zone du bois de compression.
\end{abstract}

\section{Introduction}

Nous examinerons ici l'influence de la fertilisation sur le bois normal et le bois de compression chez le pin maritime.

Le bois de compression, qui est le bois de réaction des gymnospermes, a fait l'objet de très nombreuses études, comme par exemple : FuIITa et al. (1978), Harris (1977), Nicholls (1982), Ohta (1979), Polge \& Illy (1967), Seth (1979), Thimell (1979, 1980, 1982). 
La présence de ce bois constitue un défaut pour l'utilisation. C'est un type particulier de bois, conséquence d'une réponse physiologique de l'arbre à diverses sollicitations extérieures (géotropisme, phototropisme, concurrence, vents dominants...) ou peut-être intérieures (contraintes de croissance...).

Dans le cas du pin maritime, il semble que les vents dominants d'Ouest jouent un rôle important dans la formation du bois de compression du côté Est des arbres.

Sa présence a des répercussions sur le nombreuses propriétés du bois, couleur, rétractibilité, largeur de cernes, excentricité, densité, composition chimique, et donc sur son utilisation, que ce soit comme bois d'œuvre ou comme matière première pour l'industrie papetière.

\subsection{Echantillonnage}

On a tiré une rondelle d'un centimètre d'épaisseur dans chacun des 172 arbres échantillons décrits dans la $1^{\text {r" }}$ partic (paragraphe 21), à la hauteur de $1,30 \mathrm{~m}$; sur chaque rondelle on a délimité les 5 années de 1966 à 1970, la saison de végétation 1966, année à partir de laquelle apparaît le bois de compression, étant la $10^{\circ}$ année de croissance depuis le semis.

Compte tenu de cette précaution, les comparaisons deviennent possibles pour les zones de bois normal ou de compression prises séparément ou regroupées.

Plusieurs directions ont été étudiées afin de mettre en évidence une différence éventuelle dans les propriétés du bois normal et du bois de compression.

\subsection{Définition des zones de bois par rapport au bois de compression}

La figure 1 indique la définition des 4 zones dans chaque rondelle-échantillon. ainsi que la terminologie adoptéc, qui sera utilisée dans ce qui suit ; elle schématise également l'emplacement des 5 années étudiées. L'un d'entre nous a étudié en détail la structure de ces 5 accroissements annuels (OHTA, 1979, op. cit.).

Le bois de compression occupe le secteur 3, centré autour de $0^{\prime \prime}$. Le bois normal, ou bois opposé, occupe le secteur 4 , centré autour de $180^{\circ}$. Le bois intermédiaire, qui a des propriétés a priori plutôt comparables à celles du bois normal, occupe les secteurs 1 et 2 , centrés autour de 90" et $270^{\circ \prime *}$.

\subsection{Caractères étudiés}

Sur cet échantillonnage ont été étudiées la largeur des cernes, l'excentricité de la moelle, la densité du bois et 2 caractéristiques papetières, le rendement en pâte et la longueur de fibres.

\footnotetext{
: Bois de compression - Compression nood. Bois normal, bois opposé - Opposite wood. Bois intermédiaire - Side wood.
} 


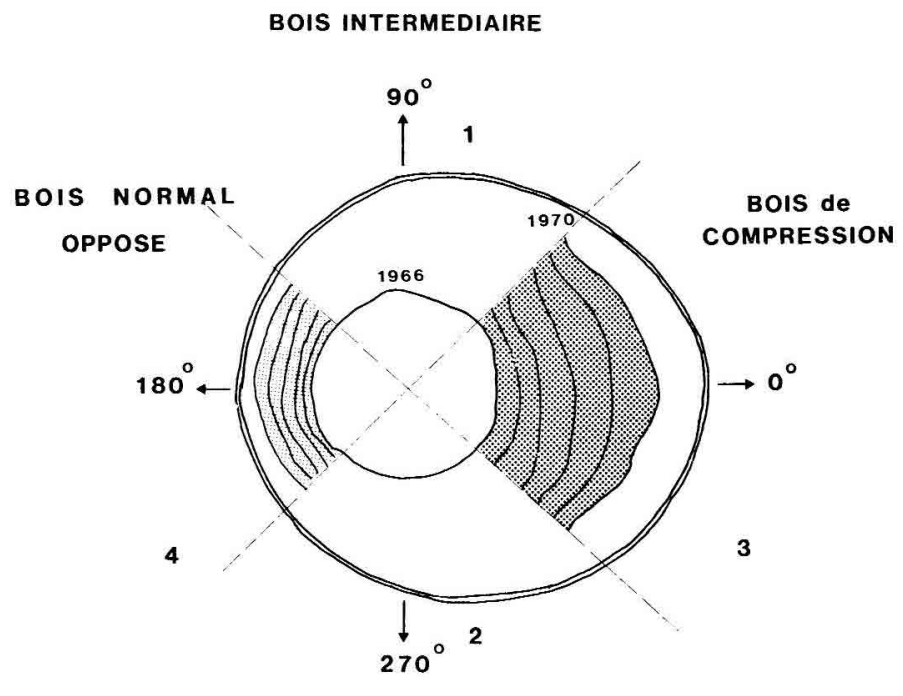

BOIS INTERMEDIAIRE
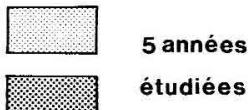

FIG. 1

Section transversale.

Cross section.

Définition des quatre zones - Definition of the four zones.

1 et 2 : bois intermédiaire - side wood.

3 : bois de compression - compression wood.

4 : bois opposé - opposite wood.

\section{Résuiltats}

\subsection{Largeur des cernes}

La vitesse de croissance radiale a déjà été évoquée à propos des billons et éprouvettes normalisées étudiés dans la première partie (paragraphes 2211 et 2221).

La figure 2 indique la fréquence des largeurs moyennes de cernes, toutes zones et tous traitements confondus.

Le tableau 1 donne les résultats des comparaisons de moyennes pour le bois normal, le bois de compression et la réunion des dcux catégories. Le groupe des traitements contenant $\mathrm{P}$ dépasse dans les 3 cas le groupe des témoins et des traitements sans $\mathrm{P}$ ( $\mathrm{N}$ et $\mathrm{NK})$. 


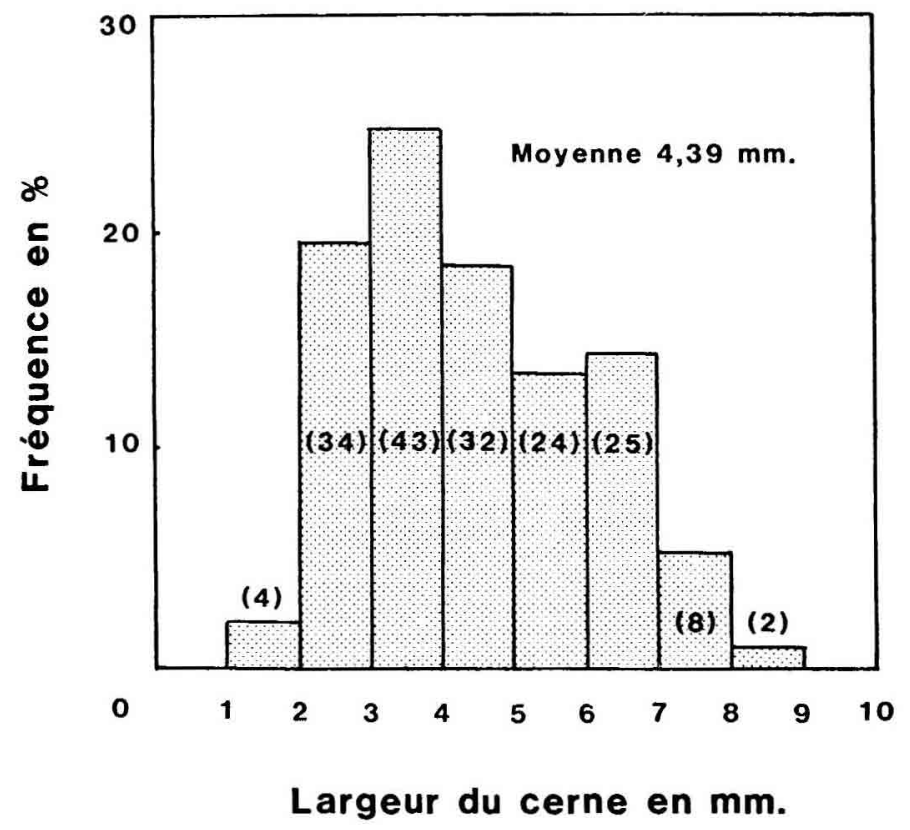

\section{$(\ldots)=$ nombre d'arbres par classe}

FIG. 2

Histogramme des largeurs de cernes.

Histogram of the annual rings width for all the trees.

Fréquence en p. 100 - Frequency, p. 100.

Moyenne - Mean.

Largeur de cerne en mm - Ring width, mm.

Nombre d'arbres par classe - Number of trees for each class.

La figure 3 montre que la largeur de cerne cumulée sur les 5 ans est supérieure dans le bois de compression, quels que soient les traitements; les points sont situés au-dessus de la première bissectrice et l'on notera la séparation entre les groupes avec $P$ et les groupes sans $P$.

\subsection{Excentricité de la moelle}

On observe très souvent, dans le cas du pin maritime, une excentricité de la moelle et nous avons procédé à sa mesure pour les sept traitements. 


\section{TABleau 1}

Résultats des comparaisons de moyennes sur les largeurs de cernes de 1966 à 1970 a à $1,30 \mathrm{~m}$, ainsi que sur l'excentricité.

Results of tests of comparisons of means for ring width from 1966 till 1970, at breast height, and for eccentricity.
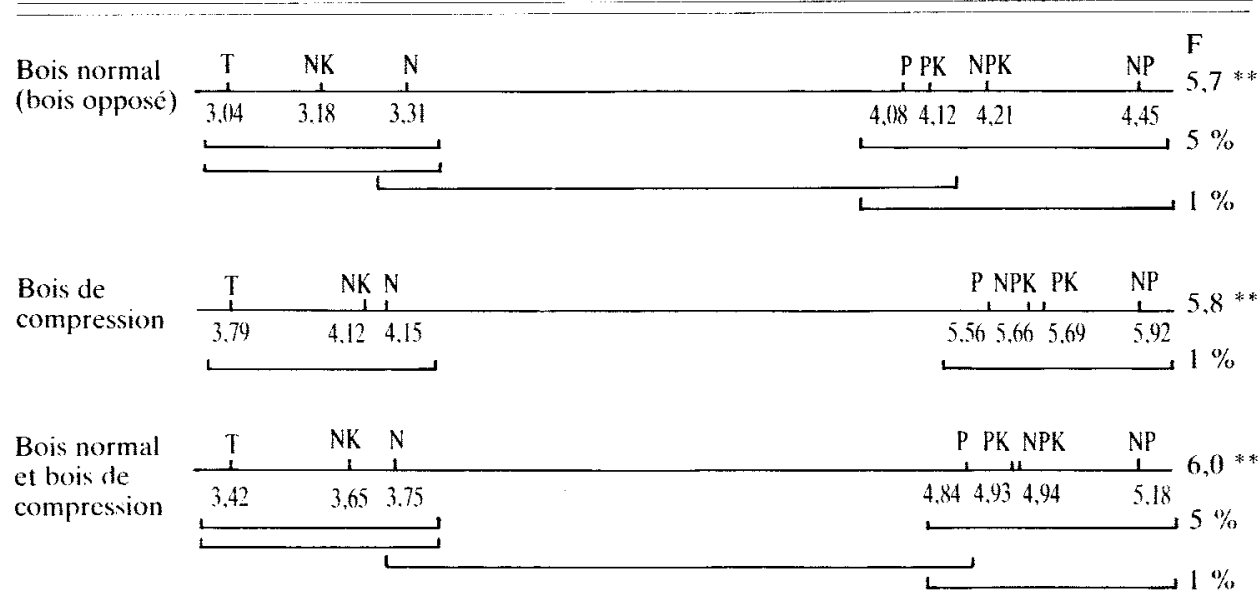

\begin{tabular}{|c|c|c|c|c|c|c|c|}
\hline \multirow{2}{*}{$\begin{array}{l}\text { Exccntricité } \\
\text { à } 1,30 \mathrm{~m}\end{array}$} & $\mathrm{~T}$ & $\begin{array}{c}\mathrm{N} \\
\mathrm{I}\end{array}$ & NK & NPK & $\underset{1}{N P}$ & $\underset{1}{N P}$ & p \\
\hline & 1.238 & 1,247 & 1.286 & 1,307 & 1,324 & 1.352 & 1.361 \\
\hline
\end{tabular}

(Ratio: $\frac{\text { radius of the log, compression wood }}{\text { radius of the log, normal wood }}$ ).

Other captions like fig. 1 .

* : test $F$ significant at 5 p. 100.

** : test $F$ significant at 1 p. 100.

$* * *$ : test $F$ significant at 1 p. 1000 .

N.S. : test $F$ not significant.

Elle est définie, dans cette étude, par le rapport $\mathrm{e}=\frac{\mathrm{Rc}}{\mathrm{Ro}} \times 100$ où $\mathrm{Rc}$ et $\mathrm{Ro}$ sont mesurés sur le diamètre $0^{\prime \prime}-180^{\prime \prime}$ de la section entière des rondelles (fig. 1).

Comme l'ont montré Polge et ILLY (1967), l'excentricité de la moelle est révélatrice de la présence de bois de compression. 


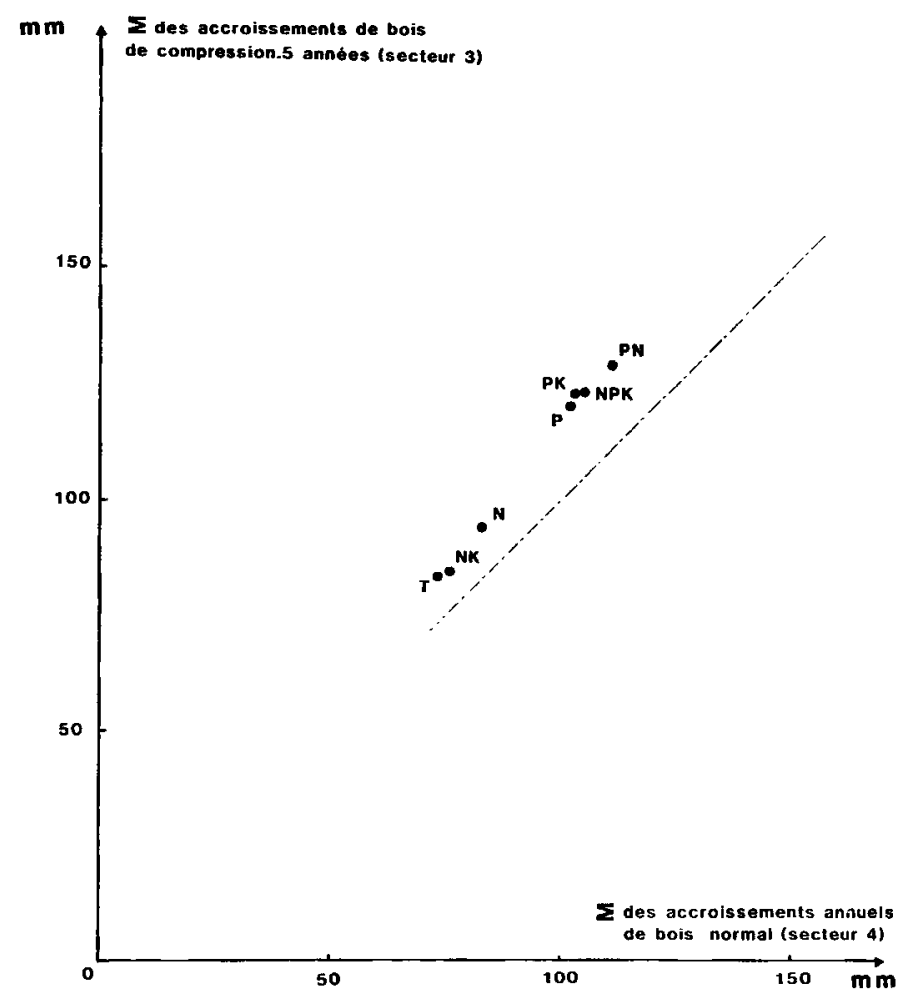

FIG. 3

Comparaison des sommes des 5 accroissements annuels pour les zones de bois normal et de bois de compression en fonction des traitements.

Comparison of the stums of the 5 annual rings between the normal wood and compression wood zones depending upon the treatments.

Accroissement radial cumulé des 5 années de bois de compression - Cumulated radial growth of the 5 compression wood years.

Accroissement radial cumulé des 5 années de bois normal - Cumulated radial growth of the 5 normal wood years. 
Cependant, dans le cas de cette expérience, lorsqu'on considère les douze années à $1,30 \mathrm{~m}$, on n'observe pas de différence significative entre les traitements pour l'excentricité (tabl. 1), bien que les résultats relatifs aux largeurs des 5 cernes montrent des différences entre traitements. Ce résultat est donc différent de celui qui a pu être annoncé dans la première partie (paragraphe 2213) à partir de mesures faites à $0,30 \mathrm{~m}$.

La fertilisation ne semble donc pas entraîner un défaut de forme systématique, en section transversale.

L'excentricité est, bien entendu, une conséquence de la présence de bois de compression. Dans une publication récente, l'un de nous (OHтA, 1979, op. cit.), à partir des mêmes échantillons que ceux dont il est question ici, indique le nombre d'individus comportant du bois de compression dans les 7 modalités de traitement. Le phénomène de l'apparition de ce bois de réaction y est étudié en fonction de la distance par rapport à la moelle de points des divers rayons des rondelles $\left(0^{\prime \prime}, 90^{\prime \prime}\right.$, $180^{\circ}$ et $\left.270^{\prime \prime}\right)$ tels que nous les avons définis au paragraphe 12 .

\subsection{Densité du bois}

L'infradensité du bois a été mesurée pour chaque rondelle suivant les 4 directions définies au paragraphe 12 (fig. 1), sur les 5 années de 1966 à 1970 (tabl. 2).

\subsubsection{Zone de bois de compression}

L'analyse des résultats montre une assez faible différenciation statistique entre les traitements ; seul le traitement $\mathrm{N}$ semble se distinguer d'une partie des autres pour ce bois de compression dont la nature particulière masquerait, en quelque sorte, l'effet des divers modes de fertilisation.

\subsubsection{Zone de bois normal}

Pour le bois normal, diamétralement opposé au précédent dans la rondelle, on observe une plus forte valeur du test $F$ de SNEDEcor $(F$ observé $=5,3)$ et donc des différences plus nettes entre les moyennes de traitements.

Cette fois-ci, le traitement témoin $\mathrm{T}$ se détache de tous les traitements fertilisés. En outre, l'ensemble des traitements présente, en moyenne, une densité inférieure d'environ $20 \mathrm{~g} / \mathrm{dm}^{3}$ à celle du bois de compression, ce qui correspond bien aux propriétés connues du bois de compression des résineux.

\subsubsection{Zone de bois intermédiaire}

La répartition des résultats ressemble beaucoup à celle du bois normal, en particulier avec la plus forte valeur pour le témoin. Les traitements contenant de l'azote présentent les plus faibles valeurs, NK et $P$ étant intermédiaires.

\subsubsection{Bois des quatre zones confondues}

Les résultats sont tout à fait analogues au cas précédent; c'est en moyenne, la valeur de la densité globale de chaque rondelle. Notons encore la forte valeur de la densité des témoins et la place de transition des traitements NK et P. 
TABLEAU 2

Résultats des mesures de densité sur les cinq années (1966 à 1970).

Results of measurements of wood density for the five years (from 1966 till 1970).

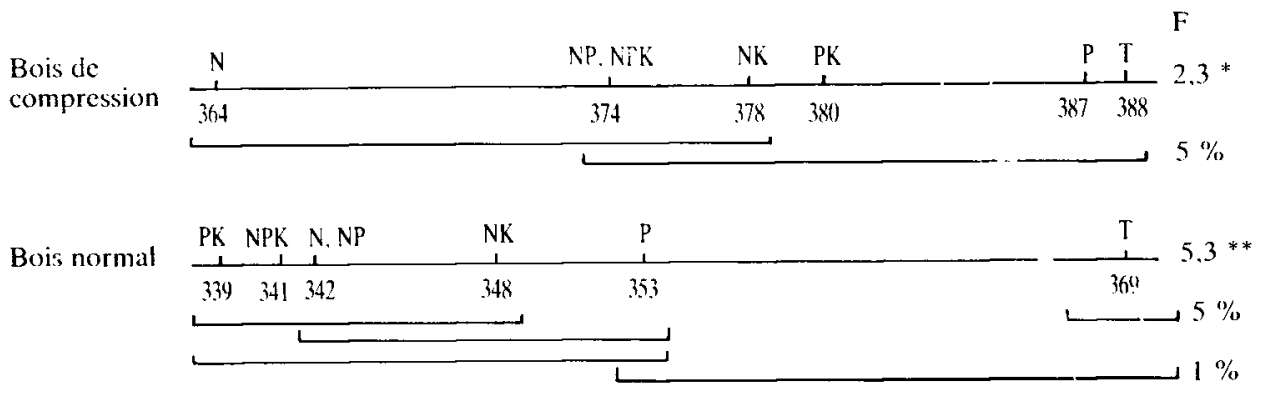

Bois

intermédiaire
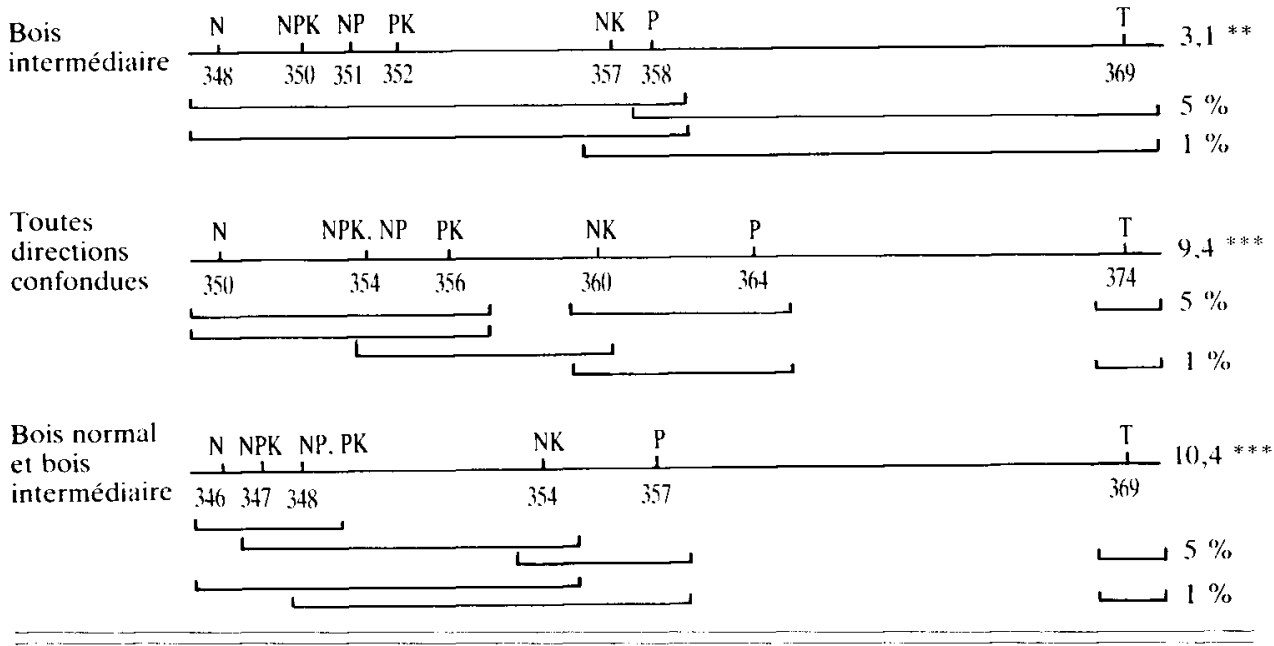

\subsubsection{Zones du bois normal et du bois intermédiaire}

Le calcul effectué sur l'ensemble des 3 directions ne comportant pas de bois de compression montre encore, de façon très nette, le regroupement des traitements fertilisés, dont la densité varie dans un domaine assez étroit, de $346 \mathrm{~g} / \mathrm{dm}^{3}$ à $357 \mathrm{~g} / \mathrm{dm}^{3}$, et la séparation du traitement $\mathrm{T}$, dont la densité est $369 \mathrm{~g} / \mathrm{dm}^{3}$, de tous les autres traitements.

En résumé, pour les directions exemptes de bois de compression, qu'elles soient prises isolément ou regroupées, on constate que :

- le traitement témoin, avec la plus forte valeur de densité, se détache de tous les autres traitements fertilisés;

- les traitements fertilisés sont groupés dans un domaine de densités assez étroit ; 
les traitements comportant de l'azote, à l'exception de NK dont les accroissements annuels ne sont pas significativement différents de ceux des témoins (voir paragraphe 21 et tableau 1), présentent souvent les plus faibles valeurs de densité, à l'écart par rapport au témoin ne dépassant pas $30 \mathrm{~g} / \mathrm{dm}^{3}$, c'est-à-dire environ 8 p. 100 ;

- l'effet dépressif de la fertilisation, de l'azote en particulier, sur la densité, cst net au sens statistique, mais somme toute assez limité sur le plan pratique ;

- le traitement $P$ se maintient à un niveau de densité intermédiaire entre lo témoin T et les autres traitements; il n'est inférieur au témoin que d'environ 4,5 p. 100.

Pour la direction contenant le bois de compression, il semble que les différences précédentes soient masquées par le phénomène «bois de réaction » au point que, à l'exception du traitement $\mathrm{N}$, on ne puisse plus faire de différence entre le traitement témoin et les traitements fertilisés; en particulier, la valeur moyenne du traitement $P$ est presqu'identique à celle du témoin.

Dans le cas où l'on examine toutes les directions confondues, les valeurs de la densité sont légèrement supérieures à celles du bois normal strict et la différenciation se fait entre traitements fertilisés, notamment ceux qui contiennent de l'azote et témoin.

Malgré un certain nombre de différences, en particulier en ce qui concerne NK, les résultats obtenus sur ces années particulières sont assez bien cohérents avec ce qui a été trouvé à partir des éprouvettes normalisées d'essais mécaniques (première partie, paragraphe 222 et tableau 4) : regroupement des traitements fertilisés contenant $N$, ou $\mathrm{N}$ et $\mathrm{P}$, vers les densités plus faibles, place intermédiaire de $\mathrm{P}$ ou de $\mathrm{PK}$.

Le traitement NK ne présente pas d'intérêt particulier, étant donnée sa vitesse de croissance radiale aussi faible que celle des témoins non fertilisés.

\section{Influence de la fertilisation sur les caractéristiques papetières}

Les propriétés papetières du bois les plus importantes sont le rendement en pâte et la longueur moyenne des fibres (trachéides). On se propose de les étudier successivement, la première en rapport avec la densité du bois et la seconde en fonction de l'âge, pour les différentes directions définies au paragraphe 12 .

\subsection{Rendement en pâte}

\subsubsection{Echantillonnage}

A partir des 172 arbres étudiés précédemment (OнтA, 1979, op. cit.), nous avons constitué un deuxième échantillonnage restreint; pour chacun des 7 traitements, nous avons retenu 6 arbres, suivant 4 directions, soit 168 échantillons de bois pris radialement sur lesquels ont été déterminés à la fois l'infradensité et le rendement en pâte ; ces arbres présentaient une densité moyenne égale à celle du traitement auquel ils appartenaient. 

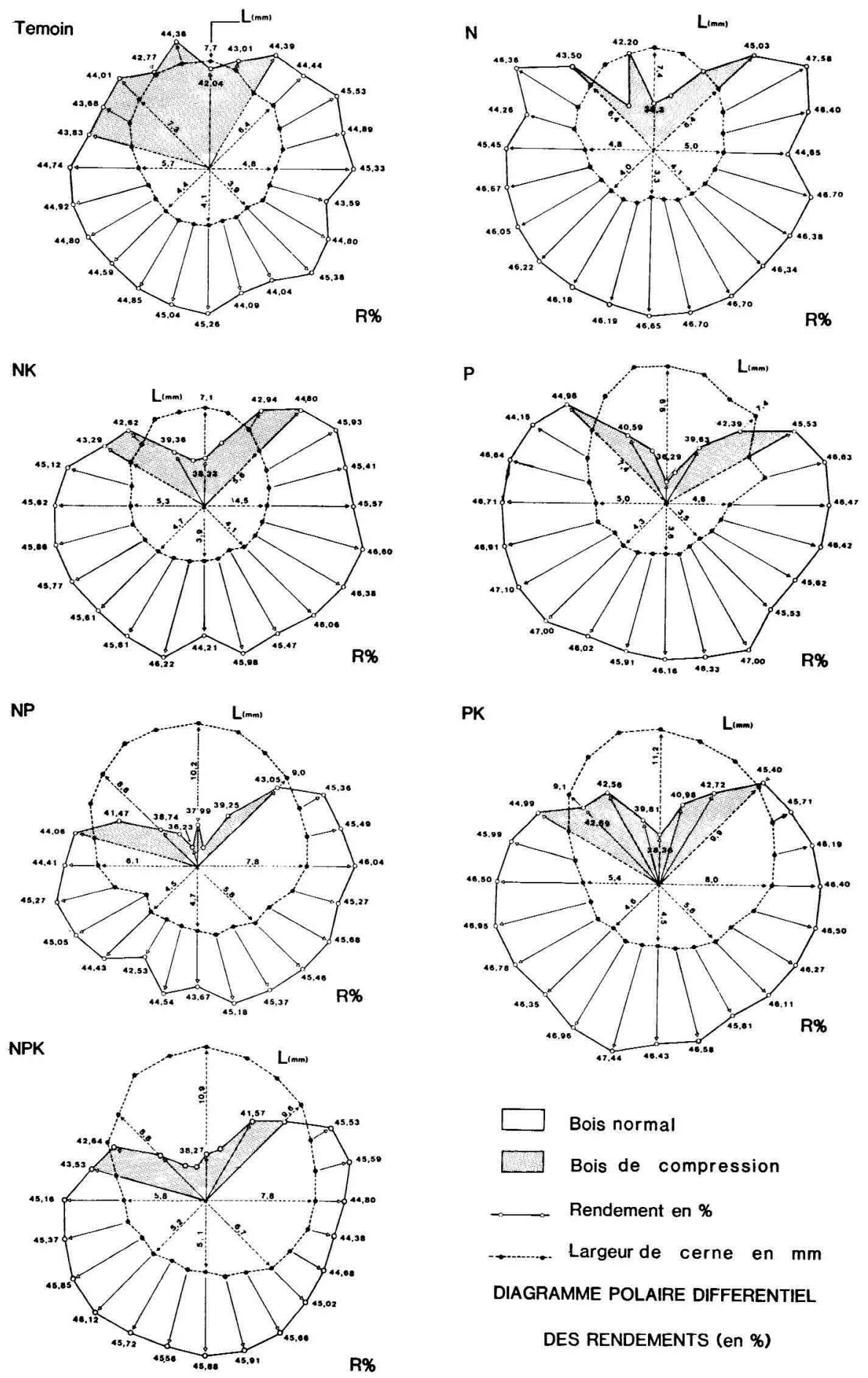

$\square$ Bois de compression

Rendement en \%

........ Largeur de cerne en $\mathrm{mm}$

DIAGRAMME POLAIRE DIFFERENTIEL

DES RENDEMENTS (en \%)

FIG. 4 
La présence de bois de compression (direction 3), dont le comportement se distingue fortement de celui des autres directions (paragraphe 1), nous a amenés à considérer le cas pratique où les 4 directions sont représentatives de l'arbre entier, puis les cas où l'on simule l'élimination du bois de compression en ne retenant que les directions $1,2,4$, réunies, combinées deux à deux ou isolées.

Dans chaque traitement, le choix de l'arbre moyen a permis d'établir le diagramme polaire des rendements (JANIN, 1972, 1980) en prélevant des échantillons radiaux suivant 24 directions (fig. 4 ).

\subsubsection{Méthodes}

Le rendement en pâte a été déterminé en microlessiveurs (JANIN, 1981) à l'aide du procédé KRAFT $\left(\mathrm{NaOH}+\mathrm{Na}_{2} \mathrm{~S}\right)$ dans les conditions suivantes :

- alcali actif 20 p. 100 ;

- sulfidité 25 p. 100 ;

- rapport $\frac{\text { liquide }}{\text { bois }}: 4$;

- diagramme température-temps : 1 h 30 de $20^{\circ} \mathrm{C}$ à $170^{\circ} \mathrm{C}, 1 \mathrm{~h} 30$ à $170^{\circ} \mathrm{C}$.

Les autres caractères mesurés sont la largeur des cernes, l'infradensité et le produit infradensité $\times$ rendement en pâte qui exprime le poids de fibres anhydre à l'état écru, utilisable par l'industrie papetière, rapporté au volume des arbres sur pied (JANIN, 1983).

\subsubsection{Résultats}

\subsubsection{Analyse de la variance}

Les résultats de l'analyse de variance sont consignés dans le tableau 3, colonnes 1 à 13. Les effets considérés sont l'influence de la fertilisation et des directions.

\section{Facteur fertilisation}

- Sur le rendement en pâte

Il n'y a qu'un effet faiblement significatif $(F=2,5)$ lorsque le bois de compression est associé aux 3 autres directions (tableau 3, colonne 2, cas réel de l'approvisionnement des industries de pâte) ; l'effet n'est pas significatif quand il est associé à l'une quelconque d'entre elles (colonnes $7,8,9$ ).

\section{Diagrammes polaires différentiels des rendements en pâte et des largeurs de cernes des 5 années étudiées.}

Differential polar diagrams of pulp yields and radial growth of the 5 examinated years.

Témoin - Control.

Rendement en p. 100 - Pulp yield, p. 100.

Other captions like fig. 1 . 


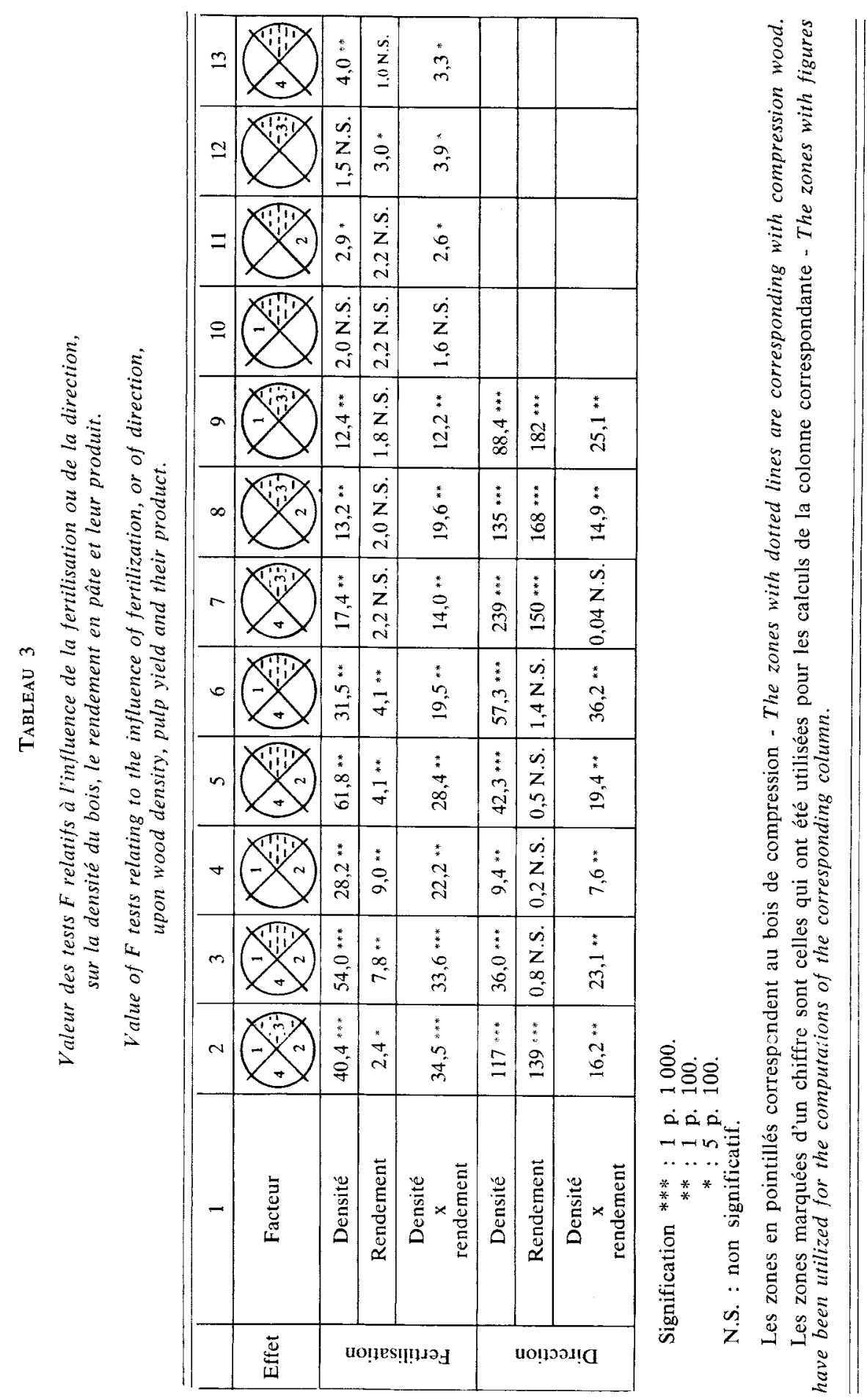


En revanche, un effet plus net apparaît $(F>4)$ lorsque les trois directions de bois normal ou intermédiaire sont regroupées (colonnes $3,4,5,6$ ) ou lorsqu'on ne considère que le bois de compression (colonne 12, $\mathrm{F}=3,0$ ).

Notons que lorsque le bois de compression est pris seul en compte, le rendement diminue quelles que soient les modalités de fertilisation et d'autant plus, semble-t-il, qu'elles contiennent du phosphore (tabl. 4).

\section{TABLEAU 4}

Résultuts des comparaisons de moyennes sur les rendements en pâte. correspondant aux colonnes 2, 3 et 12 du tableau $j$.

Results of tests of comparisons of means for pulp yields, corresponding to 2 nd, 3 rd and 12 th columns of 3 rd table.

Quatre

directions

confondues

\begin{tabular}{|c|c|c|c|c|c|c|}
\hline $\mathrm{N}$ & $P$ & $\mathrm{NP}$ & PK & NPK & $\mathrm{NK}$ & $\mathrm{T}$ \\
\hline${ }^{H .3}$ & 44.4 & 4,5 & 44.7 & 45.1 & 45,2 & 45.4 \\
\hline
\end{tabular}

Trois

directions

confondues

(pas de bois

de compression)

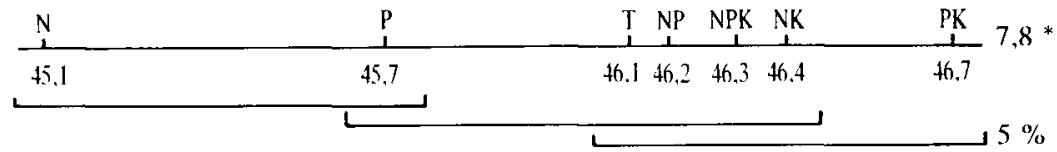

Dircction du

bois de

compression

\begin{tabular}{|c|c|c|c|c|c|}
\hline PK & $\mathrm{NP}$ & 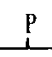 & NPK & $\mathrm{N}, \mathrm{NK}$ & $T$ \\
\hline 38.7 & 39.5 & 40.6 & 41,3 & 41,8 & 43,3 \\
\hline
\end{tabular}

Quatre directions confondues - The four zones of wood together.

Trois directions confondues (pas de bois de compression) - Three zones of wood together (no compression wood).

Direction du bois de compression - Compression wood only.

En l'absence de bois de compression, le rendement est plus élevé, et peut-être d'autant plus que les modalités de fertilisation comportent du phosphore.

- sur la densité

On observe presque toujours un effet de fertilisation; mais lorsquion considère le bois de compression seul, il n'y a pas de différences entre traitements (tableau 3 , colonne 12). Le tableau 5 résume ces observations.

- sur le rendement en pâte par unité de volume (densité $\times$ rendement)

On observe toujours, à une exception près (tableau 3, colonne 10), une différence entre les traitements. La signification au seuil de 1 p. 1000 dans le cas de l'arbre entier (tableau 3, colonne 2) est une indication très utile pour les forestiers, parce qu'elle correspond à la production de pâte par unité de volume de bois sur pied. 


\section{TABLEAU 5}

Résultats des comparaisons de movennes sur les densités correspondant aux colonnes 2,3 et 12 du tableau 3.

Results of tests of comparisons of means for wood densities, corresponding to $2 \mathrm{nd}$, 3rd and $12 \mathrm{th}$ columns of $3 \mathrm{rd}$ table.

Quatre

directions

confondues

\begin{tabular}{|c|c|c|c|c|c|c|}
\hline$P \quad N$ & PK & NPK & ${ }^{N P}$ & NK & 1 & $\begin{array}{l}F \\
40,4 \text { *** }\end{array}$ \\
\hline $347,5 \quad 349.2$ & 351,8 & 354.7 & 365.4 & 369,6 & 384.8 & \\
\hline
\end{tabular}

Trois

directions confondues

(pas de bois de compression)

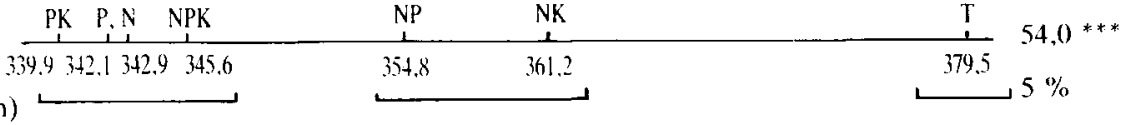

Direction du

bois de

compression

\begin{tabular}{|c|c|c|c|c|c|c|}
\hline $\mathrm{P}$ & $\mathrm{N}$ & NPK & PK & NK & $N P$ & $T$ \\
\hline 361.3 & 368,1 & $3 \times 2.0$ & 387.5 & 394.8 & 397.2 & 400.7 \\
\hline
\end{tabular}

Captions like before.

Le tableau 6 montre les effets de la fertilisation soit globalement, soit sur le regroupement des directions ne comportant pas de bois de compression, soit sur le bois de compression seul.

\section{TABILEAU 6}

Résultats des comparaisons de moyennes

sur les produits densité $x$ rendement correspondant aux colonnes 2,3 et 12 du tableau 3 .

Results of tests of comparisons of means for the products wood density $x$ pulp yield, corresponding to 2 nd, $3 \mathrm{rd}$ and $12 \mathrm{th}$ columns of $3 \mathrm{rd}$ table.

\begin{tabular}{|c|c|c|c|c|c|c|c|}
\hline $\begin{array}{l}\text { Quatre } \\
\text { directions }\end{array}$ & PN & $\mathrm{PK}$ & NPK & $\stackrel{\mathrm{NP}}{1}$ & NK & $\begin{array}{l}T \\
1 \\
\end{array}$ & $\begin{array}{l}F \\
34,5 *\end{array}$ \\
\hline confondues & $\begin{array}{lll}154.3 & 154.7 \\
\end{array}$ & 156,9 & $1.59,6$ & 162.6 & 167.1 & 174.7 & \\
\hline
\end{tabular}

Trois directions confondues (pas de bois de compression)

\begin{tabular}{|c|c|c|c|c|c|c|c|}
\hline$N$ & $\stackrel{p}{P}$ & & $\mathrm{NPK}$ & NP & $\mathrm{NK}$ & T & $33.6 * *$ \\
\hline 1.54 .3 & 156,0 & 158,4 & 159.7 & 163,6 & 167.2 & 174,9 & \\
\hline
\end{tabular}

Dircution du bois de compression

\begin{tabular}{|c|c|c|c|c|c|}
\hline p & PK & N & NPK. NP & $\mathrm{NK}$ & $\mathrm{T}$ \\
\hline 149.2 & 152.4 & 155,9 & $159,3159.6$ & 166.8 & 174.1 \\
\hline
\end{tabular}

Captions like before. 


\section{Facteur direction}

- sur le rendement en pâte

Dans le cas de larbre enticr (4 directions) on obscrve de fortes différences entre les directions, significatives au seuil de 1 pour $1000(F=139)$ dues principalement à la présence de bois de compression dans l'analyse (tableau 3, colonne 2). En effet, en l'absence de bois de compression (colonne 3), il n'y a pas de différence entre les 3 directions ( $F=0,8$ N.S.).

Les résultats des colonnes 4,5 et 6 confirment cette observation : aucune différence significative n'apparait entre les directions, autres que celle du bois de compression, prises 2 à 2 (F de 0,$2 ; 0,5$ et 1,4 ; tous N.S.).

En revanche, dès que le bois de compression est comparé à l'une des 3 autres directions, les différences sont très hautement significatives $(F>150)$.

\section{TABLEAU 7}

Résultat des comparaisons de moyennes sur les rendements en pâte (p. 100) correspondant à la colonne 2 da tablea 3.

Results of tests of comparisons of means for the pulp yield (p. 100), corresponding to 2 nd column of 3rd table.

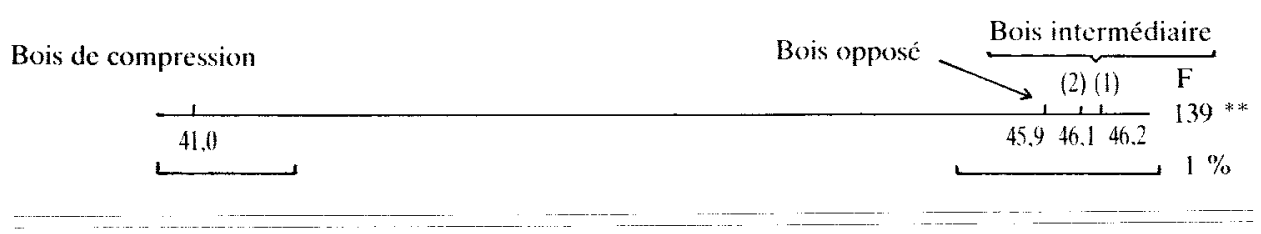

- sur la densité

On trouve constamment des différences entre directions, quelles que soient les comparaisons entreprises (tableau 3, colonnes 2 à 9). Lorsque le bois de compression entre dans la comparaison, il augmente la différence.

- sur le rendement en pâte par unité de volume

Le produit densité $\times$ rendement $\left(\mathrm{kg}\right.$ de fibres par $\mathrm{m}^{3}$ de bois) montre des effets de lorientation toujours significatifs à 1 p. 100 , sauf dans la comparaison faisant intervenir le bois de compression et le bois opposé (tableau 3, colonne 7); ces directions représentent des cas extrémes comme le montre le tableau 8.

Ceci correspond, dans l'analyse de variance, à un très fort effet de la direction sur la densité $(F=239)$, sur le rendement en pâte $(F=150)$, et à un effet nul ( $F=0,04$ N.S.) sur le produit rendement $\times$ densité. C'est un des exemples les plus évidents de compensation entre le rendement (faible) et la densité (forte) qui masque l'effet de la direction dans l'arbre entier. 
TABLEAU 8

Influence de la nature du bois (bois normal, bois de compression) sur le rendement en pate.

Influence of the wood character (normal wood, compression wood) upon the pulp yield.

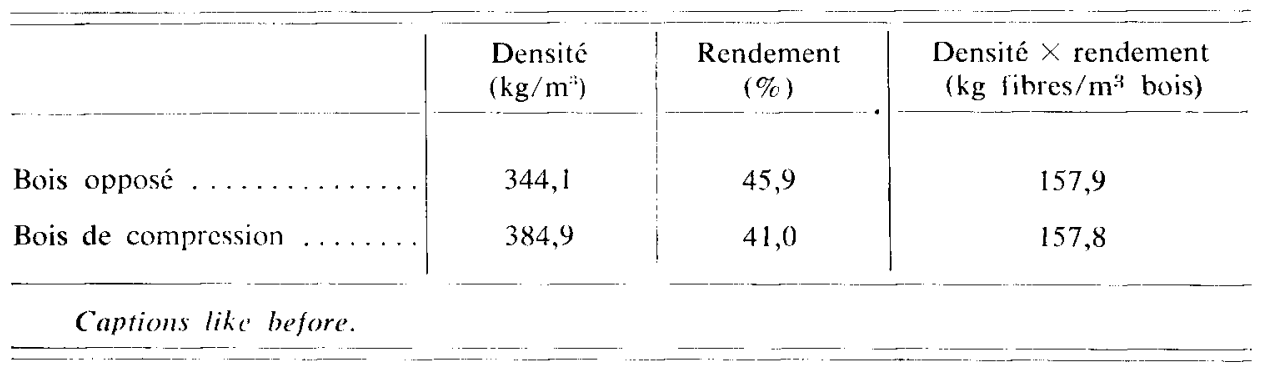

Un phénomène comparable a été mis en évidence par Petroff et Tissot (1981) sur Pinus caribaea où, sur 202 échantillons, une liaison négative a été observée entre le rendement nct en pâte et le pourcentage de bois de compression mesuré sur une section transversale des arbres $(r=-0,56$ pour 200 degrés de liberté).

\subsubsection{Principales valeurs moyennes}

Des valeurs moyennes ont déjà été données au cours de l'analyse de variance (tableaux 4 à 7 des tests de Duncan).

Les résultats qui nous semblent les plus importants ont été regroupés dans les tableaux suivants qui proviennent de la mesure, sur chaque échantillon, de la densité et du rendement en pâte : dues ;

- tableau 9, pour les 7 traitements de fertilisalion, les directions étant confon-

- tableau 10, pour les 7 traitements de fertilisation, les 3 directions ne comportant pas de bois de compression étant confondues ;

- tableau 11, pour les directions prises isolément et regroupées, tous traitements confondus.

\subsubsection{Relations entre le rendement et la largeur des cernes}

La largeur de cernes a été étudiée en détail par l'un de nous (ОнтА, 1979. op. cil.) ; l'orientation et la présence de bois de compression jouent un rôle marqué sur cet indicateur important de la qualité du bois.

OHт montre également les relations entre l'accroissement cumulé sur le rayon, suivant 24 directions, et l'infradensité : la figure 5 représente cette liaison dans le cas du traitement NPK. 


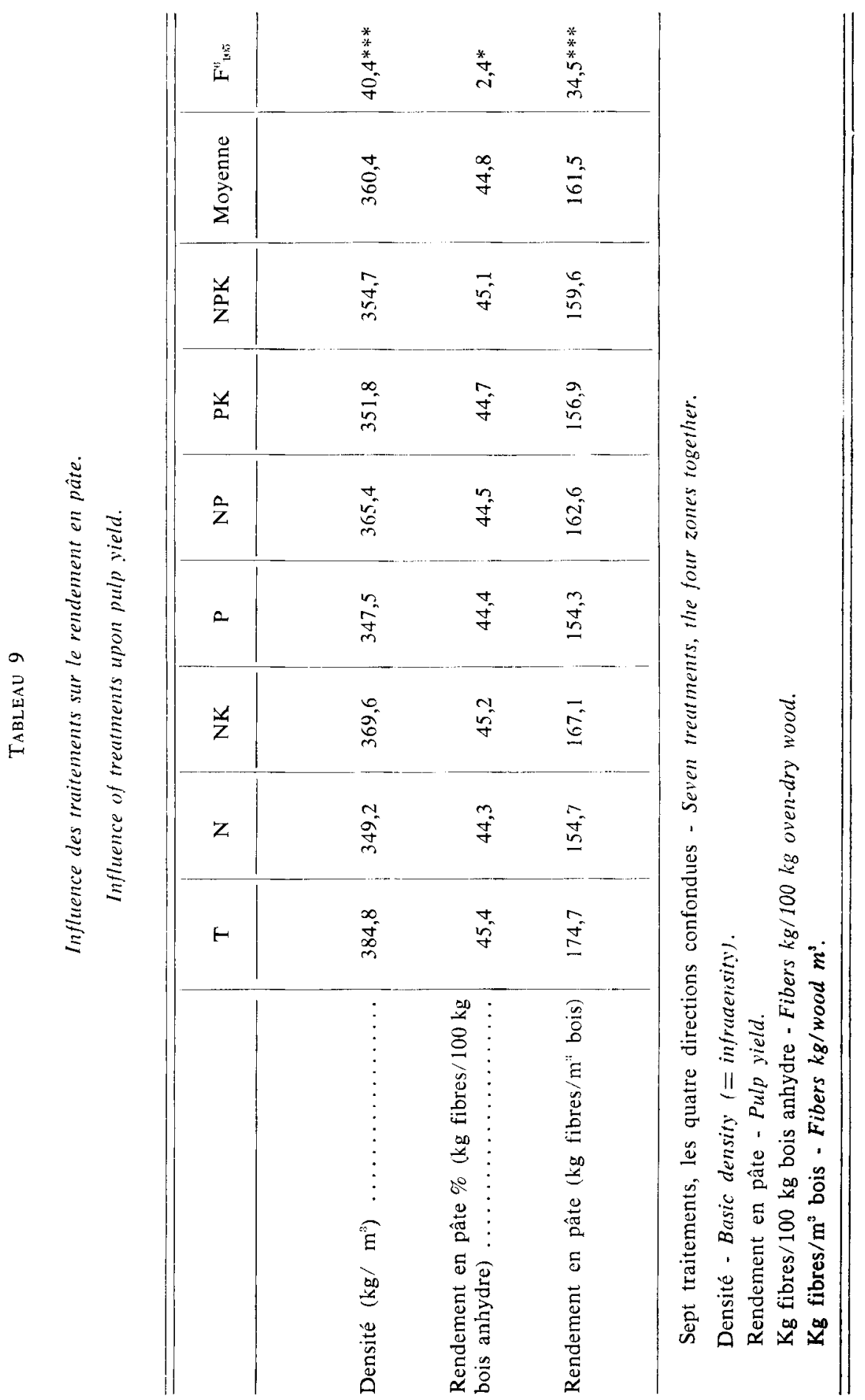




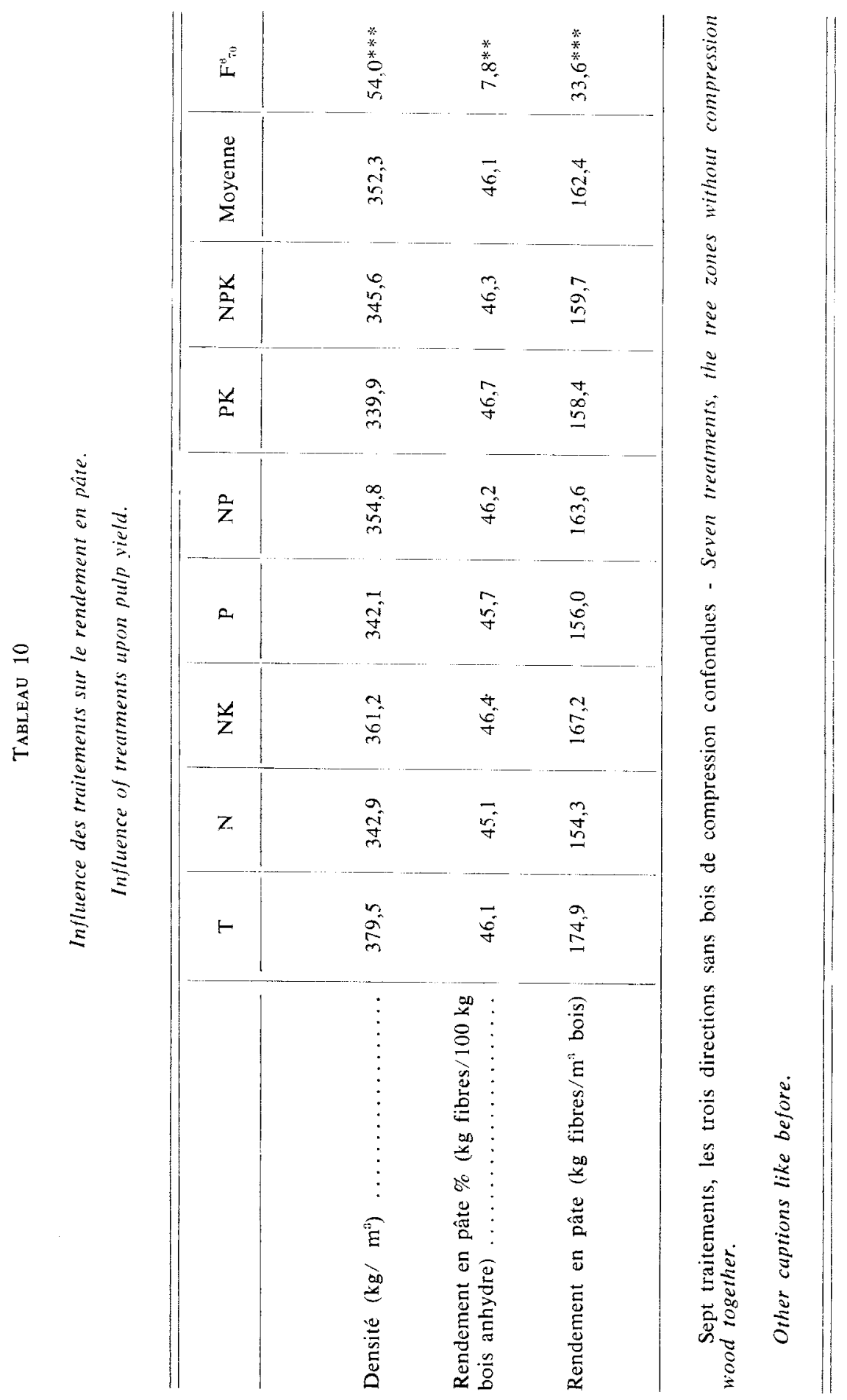




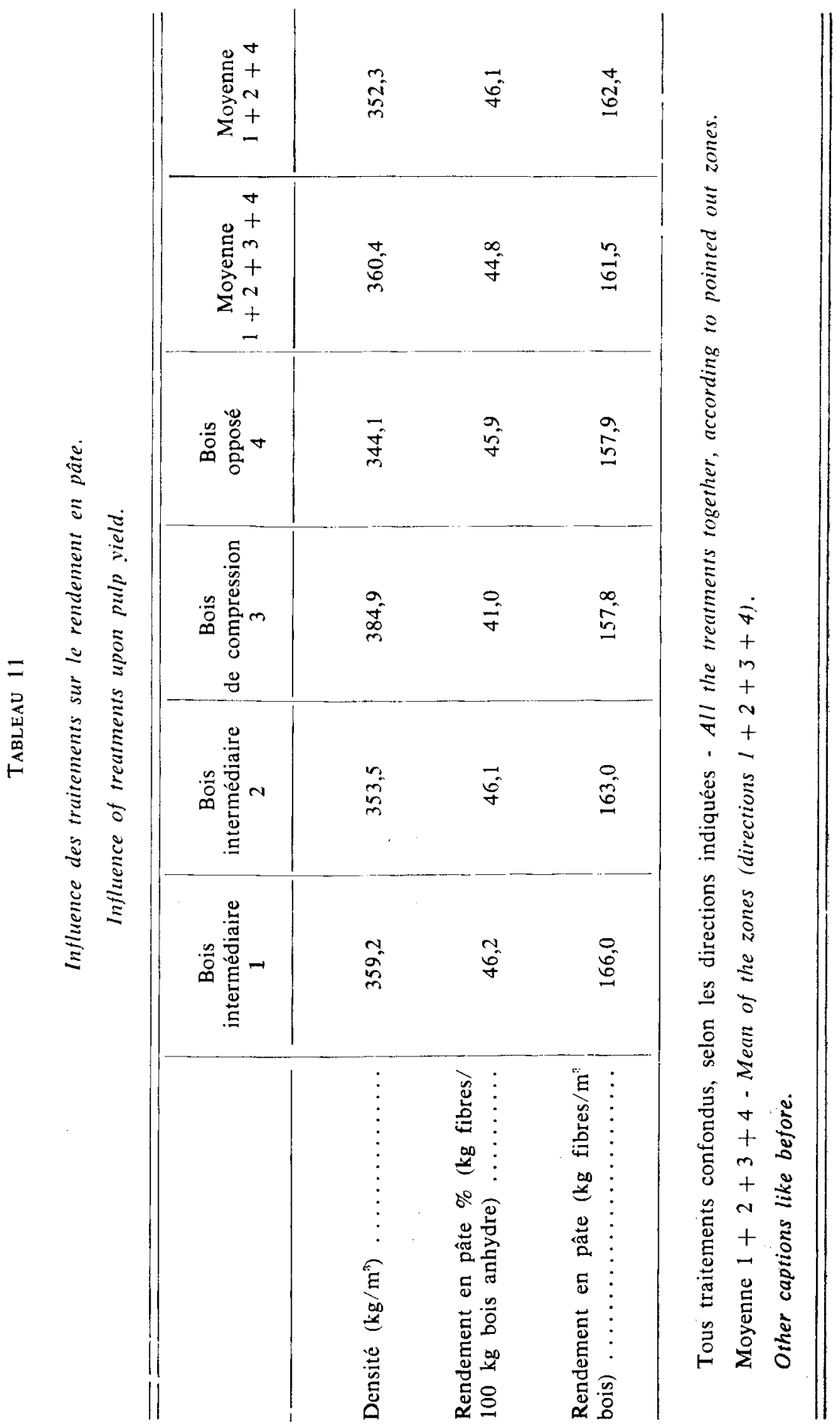




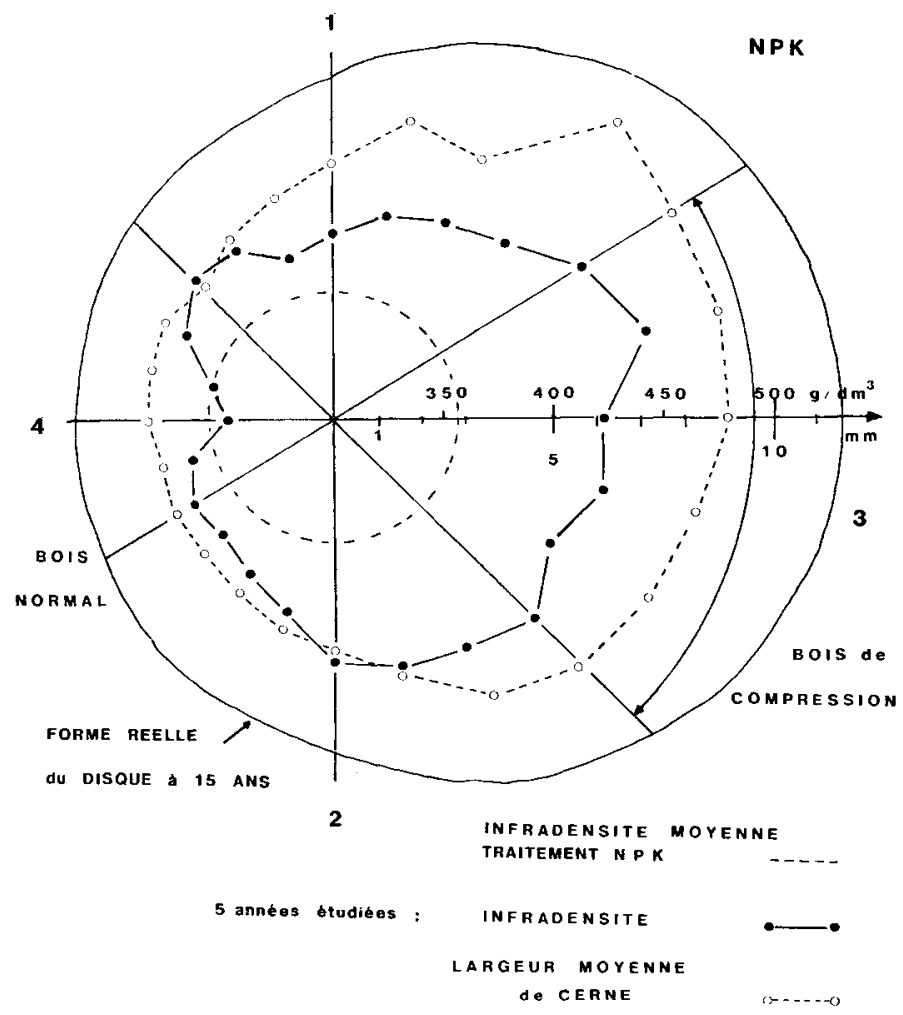

FIG. 5

Traitement NPK: section transversale montrant, pour 24 directions, les valeurs moyennes de l'infradensité et des largeurs de cernes.

$N P K$ fertilizing treatment, cross section showing, for 24 directions, the mean values of infradensity and ring width.

L'étude des diagrammes de la figure 4 fait apparaitre une diminution caractéristique du rendement, pour les directions situées dans la zone du bois de compression (secteur 3 ) et une relative homogénéité pour les autres directions.

On constate l'opposition existant entre l'accroissement cumulé sur le rayon et le rendement, à la fois à l'intérieur de chaque traitement (diagrammes polaires) et entre traitements.

La figure 6 représente, pour les traitements de fertilisation, la corrélation $(\mathrm{r}=-0,930$, signficatif à 1 p. 100) entre la diminution du rendement, dR p. 100 , du bois de compression par rapport au bois normal et l'augmentation de la largeur moyenne de cerne $\mathrm{dL} \mathrm{mm}$. 


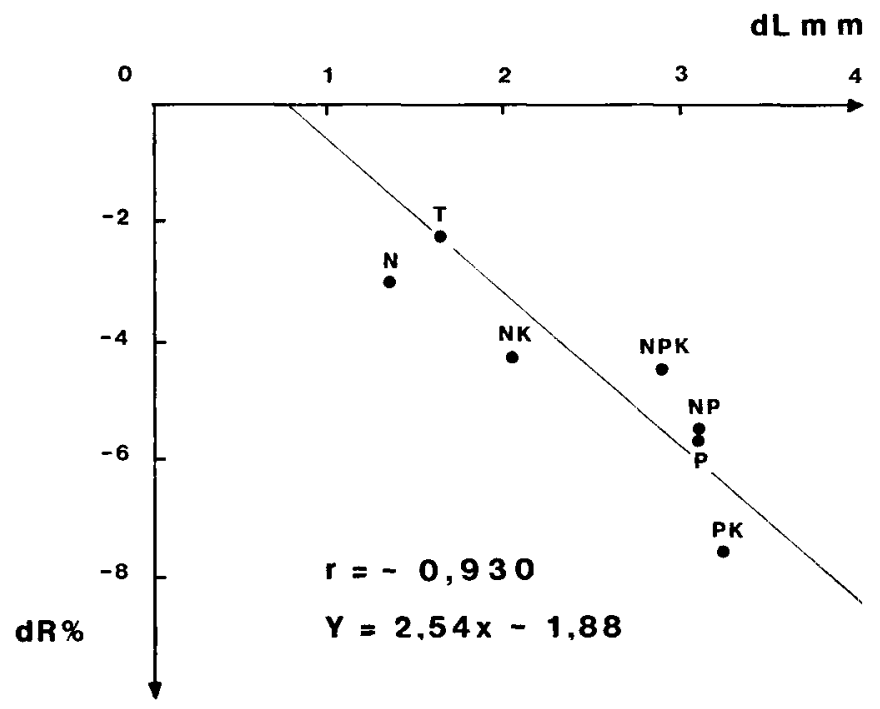

FiG. 6

Corrélation entre la diminution da rendement én pâte alR p. 100 et laugmentation de la largeur de cerne $\mathrm{dL} \mathrm{mm}$ du bois de compression par rapport au bois normal pour les 7 tratements.

Correlation between the palp yield decrease $d R$ p. 100 and the ring width increase $d L \mathrm{~mm}$ of compression wood compared with nomal wood for the 7 treatments.

\subsection{Longueur de Jibres}

\subsubsection{Echantillonnage}

Parmi les traitements de fertilisation nous avons retenu la fertilisation la plus complète, NPK, et le témoin :

- 5 arbres de chacun de ces traitements;

- 2 directions par arbre, bois de compression et bois opposé ;

- 12 années par direction, de la moelle à la périphérie.

Ces 240 échantillons ont été défibrés par microcuisson et 25 fibres ont été mesurées sur chacun d'entre eux, soit au total 6000 fibres.

\subsubsection{Résultats}

L'analyse de variance a porté, toutes années confondues, sur :

- les individus de chaque traitement;

- les directions à l'intérieur de chaque traitement ;

- les traitements à l'intérieur de chaque direction. 


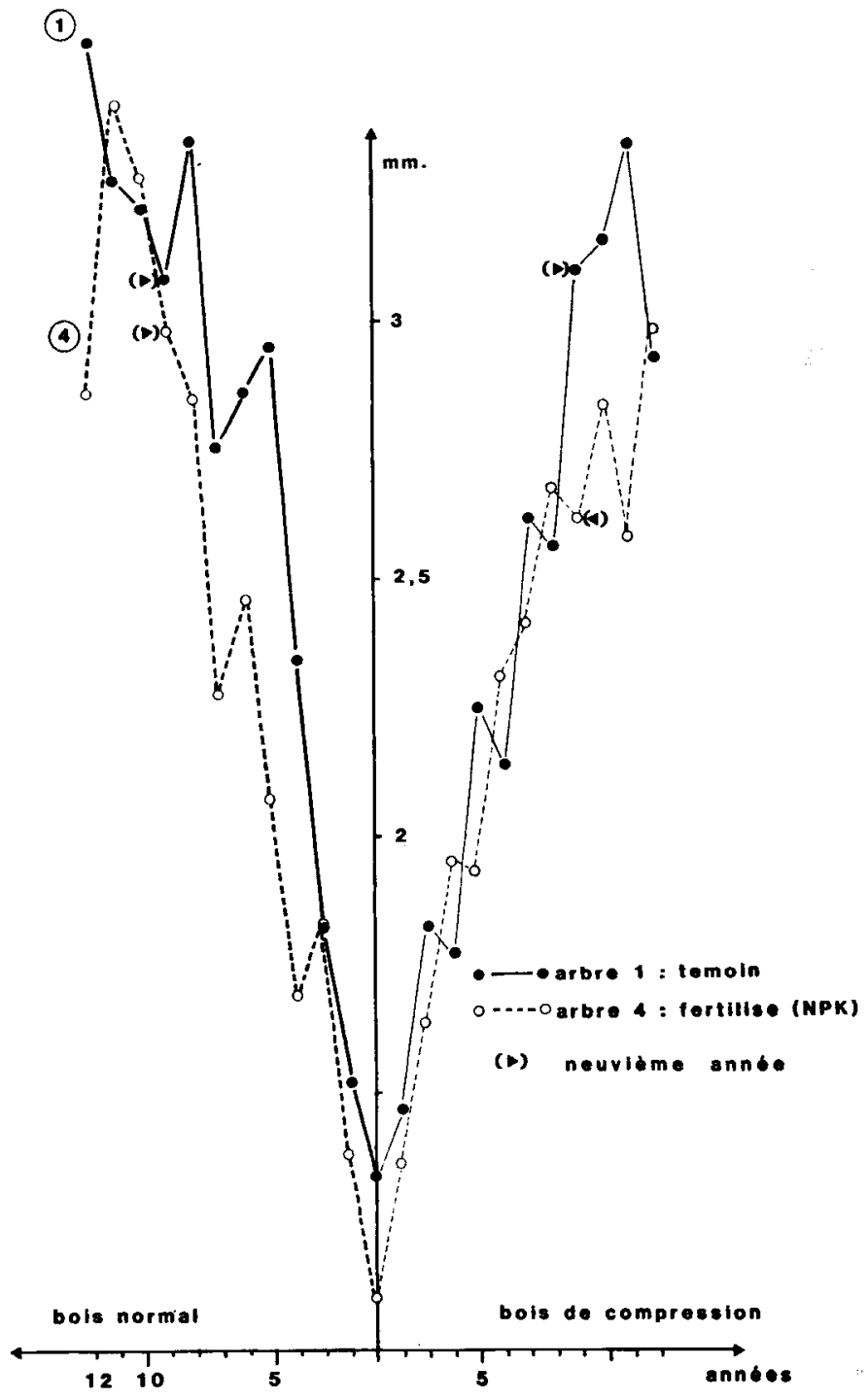

FIG. 7

Variation des longueurs moyennes de fibres par année pour le bois normal et le bois de compression.

Mean filer length : annual variation for normal wood and compression wood.

Arbre 1 : témoin - Tree 1: control tree.

Arbre 4 : fertilisé (NPK) - Tree 4: fertilized tree (NPK).

Ncuvième annéc - $9 t h$ year.

Années - Years. 
Un test s’est révélé être significatif : la seule différence qui se manifeste est relative à la comparaison entre individus pour le témoin $\left(\mathrm{F}^{+} 11: 3=3,93\right.$, significatif au seuil de 5 p. 100).

Cette remarque confirme les résultats obtenus sur le même dispositif à l'âge de 6 ans où, de plus, la fertilisation n'avait pas eu d'influence sur la longucur des fibres (Polge, 1969).

En ce qui concerne l'influence du traitoment ct de la direction, une seule différence significative apparaît dans le cas du traitement, pour la $9^{\prime \prime}$ année de croissance (fig. 7).

L'absence presque complète d'effet en fonction de la direction pourrait traduire le fait que, d'une part, toutes les années prises en compte sur la direction 3 (bois de compression) ne présentent pas, au voisinage de la moelle, l'aspect et la nature du bois de compression et, d'autre part. l'augmentation très rapide de la longueur moyenne de fibres d'une année à l'autre, variable entre les individus, rend plus difficile l'observation de différences cntre traitements dans la période juvénile.

\section{Conclusion générale}

A un moment où la ressource en pin maritime fertilisé augmente considérablement et où l'on cherche à diversifier son utilisation (par exemple fabrication des poutres lamellées et collées), il est normal de se préoccuper d'une évolution éventuelle de sa qualité liée aux nouvelles techniques sylvicoles et à la fertilisation.

En ce qui concerne ses propriétés physiques et mécaniques, la fertilisation provoque effectivement un certain nombre de modifications, quantitatives et qualitatives.

11 ressort de cette étude, sur des arbres âgés de quinze ans, que les traitements faisant intervenir l'azote en association avec d'autres éléments ont provoqué une baisse sensible des propriétés mécaniques. Du reste, on a toujours constaté un effet dépressif et l'azote utilisé seul, même par rapport au témoin et ce pour un grand nombre de caractéristiques.

En revanche, les traitements au phosphore, ou au phosphore et potassium présentent une bonne vitesse de croissance, tout en conservant une densité du bois et des propriétés mécaniques satisfaisantes, généralement non significativement différentes de celles du traitement témoin, mais avec une productivité incomparablement plus élevée. Malvos \& Bailly (1980) ont fait des observations analogues à propos de la fertilisation de Pinus khasya et de Pinus patula à Madagascar.

Les différences entre traitements sont bien marquées dans le bois pris en dehors de la zone de bois de réaction (bois de compression). Si l'on étudie l'influence du traitement sur le bois de compression, la plupart des différences observées dans le cas du bois normal disparaissent, comme masquées par cette réaction physiologique de l'arbre. 
L'examen des qualités papetières : rendement en fibres et longueur des fibres du bois de pin maritime non fertilisé et fertilisé montre qu'au niveau de l'arbre entier :

- le rendement en fibres, qui tend à augmenter avec l'âge, n'est pas influencé par la fertilisation de façon significative;

- la longueur moyenne des fibres, qui au demeurant augmente notablement d'une année sur l'autre jusqu'à l'âge de 9 à 10 ans, n'est pas modifiée par le traitement de fertilisation.

Il subsiste cependant une variabilité individuelle sensible.

Dans le détail de notre étude, nous avons montré que les combinaisons des sept traitements de fertilisation $N, P, K$ agissent différemment sur les quatre sections des arbres.

Le rendement en pâte :

- diminue pour le bois de compression et cela d'autant plus que le traitement contient du phosphore:

- augmente pour le bois opposé et le bois intermédiaire dans les mêmes cas, et cet antagonisme se traduit par une compensation au niveau des moyennes générales des traitements.

La longueur des fibres :

- n'est significativement affectée ni par la direction (bois opposé et bois de compression), ni par la fertilisation NPK, à l'âge où ont été faites nos observations.

Nous avons en effet pris la précaution de comparer les longucurs moyennes des fibres de 2 traitements T et NPK et directions (bois opposé - bois de compression) pour les années de même rang.

Ainsi, le gain de production du bois en volume obtenu au moyen de la fertilisation dans le massif des Landes demcurera malgré une légère diminution de la densité moyenne de l'ordre de 6 p. 100 lors de l'utilisation industrielle du bois et des libres, d'une part pour le rendement en pâte dans les usines de fabrication de pâte à papier et d'autre part pour la longueur des fibres lors de la mise en feuille dans les papeterics. Les données de cette étude ont été obtenues sur des arbres jeunes : il conviendrait de recommencer une étude analogue sur des arbres du même dispositif, qui scraient maintenant âgés d'environ 25 ans et plus proches de l'état adulte. 


\begin{abstract}
Summary
Effects of several fertilizers (N. P, K) on some physical, chemical, mechanical characteristics and pulp properties of maritime pine in Landes (Pinus pinaster Ait).

II. - Compression wood and pulp properties
\end{abstract}

The benefit of a volume growth oblained by fertilisers (N, P, K) for Pinus pinaster, which does not induce any important decrease of mechanical properties (fertilization with $\mathrm{P}+\mathrm{K}$, or P) as presented before (Amm. Sci. For., 1983, 40 (3), 283-298), is conserved as an advantage in pulping and paper making processes.

No practical differences in pulp qualities appear between a control and fertilised wood : i. e. for the pulp yield and fiber length.

Meanwhile detailed observations show that the wood compression zone -.. which gives by nature a low pulp yield - presents a lower pulp yield $(-4.5$ p. 100) in fertilised trees than did in control. Contrary the normal wood zones in fertilised trees has a lightly higher pulp yicld $(+0.6$ p. 100$)$ than did control.

The Pinus pinaster fiber length, which varies quite rapidly from one year to another in the young agc, is not modified by fertiliser treatments, neither between the year according to age nor in the corresponding compression wood zone.

\title{
Zusammenfassung
}

Bezichungen zwischen verschiedenen Düngungsverfahren (N, P, K) und einigen physichen, chemischen, mechanischen Eigenschaften der Scestrandkiefer (Pinus pinaster Ait.) auch in Bezug auf die Zellstoffherstellung. II. - Drackholz und Zellstoffmerkmale.

Im ersten Teil dieser Untersuchung (Ann. Sci. For., 1983, 40 (3), 283-298) wurde festgestcllt, dass die mineralische Düngung (durch N, P, K) eine Zunahme des Zuwachses nach sich zieht und zwar ohne beträchtliche Abnahme der mechanischen Eigenschaften (im Falle ciner Düngung durch $\mathrm{P}+\mathrm{K}$ oder $\mathrm{P}$ ) ; die Wirkung der Düngung ist gleicherweise bedeutsam bei der Anwendung der Seestrandkiefer für die Papierindustrie.

In Hinsicht auf die Produktion von Zelistoff oder auf die Länge der Fasern untercheiden sich die gedüngten Bäume kaum von den Kontrollbäumeñ.

Bei genaueren Beobachtungen erweist sich jedoch, dass das Druckholz der gedïngten Bäume (es liefert naturgemäss einen geringen Ertrag an Zellstoff) einen geringeren Ertrag an Zellstoff liefert $(-4,6$ p. 100) als derjenige der Kontrollbäume.

Bei allen anderen Zonen von normalem Holz der gedüngten Bäume ist der Ertrag an Zellstoff grösser $(+0,6 \mathrm{p}$. 100) als bei den entsprechenden Zonen der Kontrollbäume.

Die Länge der Fasern, die in der Jugend von Jahr zu Jahr sehr schnell variiert, wird von der Düngung nicht verändert, es sei in den Jahrringen im gleicher Alter, oder innerhalb und ausserhalb des Druckholzes. 


\section{Références bibliographiques}

Fujlita M., SAikı H., Haradi H., 1978. The secondary wall formation of compression wood tracheids. Mokuzai Gakkaishi Journal of the Japan Wood Research Society, 24 (3), pp. $158-163$.

Grlpe J., Guinaudiau J., 1974. Essai de fertilisation minérale sur pins maritimes à Mimizan (Landes). Résultats après la 16" année. Rev. For. Fr., XXVI (6), pp. 459-463.

Gelpl M., Maugi: J.P., 1973. Symposium sur la fertilisation des forêts. Livrets d'excursion. F.A.O. - I.U.F.R.O., Paris, décembre.

Gentle S.W., Bamber R.K., Humphreys F.R., 1968. Effect of two phosphate fertilizers on yield, financial yield, and wood quality of Radiata pine. For. Sci., vol. 14, n" 3, pp. 282-286.

Gunaudeau J., Illy G., Maugi J.P., Dumas F., 1963. Essai de fertilisation minérale sur pin maritime à Mimizan (Landes). Résultats après la $6^{*}$ annéc. Annales de l'Fcole Nationale des Faux et Forêts et de la Station de Recherches et Expériences, tome XX, pp. 1-72.

HARRIS J.M., 1977. Shrinkage and density of Radiata pine compression wood in relation to its anatomy and mode of formation. New Zealand Journal of Forestry Science, 7 (1), pp. $91-106$.

JANIN G. Le diagranume polaire différentiel des rendements, nouveau critère d'homogénéité des bois au point de vue des propriétés papetières. Document à usage interne. Station de Recherches sur la Qualité des Bois, I.N.R.A., C.N.R.F., n" 1972/8.

Janin G., 1981. Technique de microcuisson papetière : perlectionnement el précision des rendements en pâte. Aml. Sci. For., 38 (1), 107-126.

JANIN G., 1983. Microtests papetiers. Microcuisson, microclassage, microraffinage, mesure automatique de la longucur des fibres. Thèsé de Docteur d'Etat ès-Sciences, I.N.P.G., 5 nisi 1983

Keller R., 1968. Des caractéristiques nouvelles pour l'étude des propriétés mécaniques des bois : les composantes de la densité. Ann. Sci. For., 25 (4), pp. 237-249.

Keller R., 1973. Caractéristiques du bois de pin maritime. Variabilité el transmission héréditaire. Ann. Sci. For., 30 (1), pp. 31-62.

KEYlWERTH R., 1954. Ein Beitrag zur qualitativen Zuwachsanalyse. Die Bestimmung der Raumdichtezahl an kleinen Proben. Holz als Roh- und Werkstoff. März, pp. 77-83.

Mavge J.P., 1972. Fertilisation et croissance du pin maritime dans la région landaise. Rapport annuel AFOCEL, pp. 141-175.

Mckinnel F.H., Rudman P., Potassium fertilizer and wood density of pinus radiata. APPITA, vol. 26, n*4, janvier 1973.

Malvos C., Balley C., 1980. Expérimentations réalisées à Madagascar sur la fertilisation des boisements de pins après plantation. Bois et Forêts des Tropiques. Cahiers scientifiques $n^{\circ} 5,108 \mathrm{p}$.

MEgraw R.A., NEARN W.T., 1972. Detailed DBH density profiles of several trees from Douglas-fir fertilizer/thinnning plots. Proceedings of the symposium of the effect of growth acceleration on the properties of wood. Forest Products Laboratory. Forest Service U.S.D.A.

Monties B., Janin G. et al., 1980. Les polymères végétaux. Paris, Gauthier Villars, XIV, 345 p.

Nicholls J.W.P., 1982. Wind action, leaning trees and compression wood in Pinus radiata D. Don. Australian Forest Research, 12, 75-91.

OrTA S., 1979. The observation of tree ring structure by soft X-ray densitometry. The effect of wind direction on growth and some properties of Pinus pinaster. Mokuzai Gakkaishi, Journal of the Japan Wood Research Society, vol, 25, n" 9, 561-566.

Parker M.L., Hunt K., Warren W.G., Kennedy R.W. 1975. Effect of thinning and fertilization on intra-ring characteristics and kraftpulp yield of Douglas-fir. Eight Cellulose Conference Syracuse, New York, May 19-23. 
Petroff G., Tissot M., 1980. Variabilité des caractéristiques papetières d'un échantillonnage de Pinus caribaea de Nouvelle Calédonie. Bois et Forêts des Tropiques, mars-aivril, n"190, pp. 31-43.

Polgi: H., 1963. Contribution à létude de la qualité du bois des principales essences résineuses exotiques utilisées dans les reboisements français. Annalles de l'Fecole Nationale des Eaux et Forêts et de la Station de Recherches et Expériences, tome XX, fascicule 3, pp. 403-467.

Polge H., 1966. Etablissement des courbes de variation de la densité du bois par exploration densitométrique de radiographies d'échantillons prélevés à la tarière sur des arbres vivants. Ann. Sci. For., XXIII, fasc. 1, pp. 1-125. Thèse de Docteur ès Sciences Appliquécs, Faculté des Sciences de Nancy.

Polge H., 1969. Influence de la fertilisation sur la qualite du bois de pin maritime. Amm. Sci. For., 26 (1), pp. 45-64.

Polgl H., II..Y G., 1967. Observations sur l'anisotropie du pin maritime des Iandes. Amn. Sci. For., 24 (3), pp. 205-231.

Rueman P., MCKinnei. F.H., 1970. Effect of fertilizers on wood density of young Radiata pinc. Australiam Forestry, vol. 34, n" 3, December.

Siddiqui K.M., Gladstone W.T., Marton R., 1972. Influence of fertilization on wood and pulp properties of Douglas-fir. Proceedings of the symposium on the effect of growth acceleration on the properties of wood. Forest Products Laboratory, Forest Service U.S.D.A.

Setu M.K., 1979. Studies on the variation and correlation among some nood characteristics in Blue Pine (Pinus. Wallichiana A.B. Jackson) These Doct. Himachal Pradesh University Simla, 171005 India.

THIMELL T.E. Formation of compression wood in Balsam fir. Holzforschung. Bd. 33, 1979, pp. 137-143, 181-191; Bd. 34, 1980, pp. 5-10.

ThImel. T.E., 1982. Recent progress in the chemistry and topochemistry of compression wood. Wood Science and Technology, 16 (2), pp. 89-I22. 